\title{
Exposure to fluctuating salinity enhances free amino acid accumulation in Tigriopus californicus (Copepoda)
}

\author{
Edward M. Goolish ${ }^{1 *}$ and Ronald S. Burton ${ }^{2}$ \\ ${ }^{1}$ Department of Biology, University of Pennsylvania, Philadelphia, Pennsylvania 19104, USA \\ ${ }^{2}$ Department of Biology, University of Houston, Houston, Texas 77004, USA
}

Accepted November 28, 1987

Summary. Intracellular concentrations of free amino acids (FAA) in the intertidal copepod Tigriopus californicus increase in response to hyperosmotic stress and decrease in response to hypo-osmotic stress. The purpose of this study was to determine if exposure to repeated bouts of osmotic stress resulted in changes in FAA accumulation or the degree of FAA retention in subsequent episodes. Five groups of $T$. californicus were exposed for 22 days to a fluctuating salinity regime which consisted of $24 \mathrm{~h}$ at $100 \%$ seawater followed by $24 \mathrm{~h}$ at either $90,80,70,60$, or $50 \%$ seawater (11 cycles). After the tenth exposure to $100 \%$ seawater, individuals from each treatment group were analyzed for alanine and proline concentration. Alanine and proline accumulation generally increased in proportion to the osmotic stress up to $60-100 \%$ seawater - additional osmotic stress failed to increase total accumulation. Prior exposure to fluctuating salinity increased the extent of alanine and proline retention observed upon transfer to a hypo-osmotic medium. The treatment group which had experienced the most extreme fluctuation $(50-100 \%$ seawater) retained alanine and proline levels approximately 10 - and 20 -fold higher, respectively, than controls. A less severe salinity fluctuation was required to elicit this response for alanine $(90-100 \%$ seawater) than for proline (60-100\% seawater). Previous exposure to fluctuating salinity also resulted in increased alanine and proline accumulation in subsequent episodes of hyperosmotic stress. $24 \mathrm{~h}$ after transfer from 50 to $100 \%$ seawater, alanine and proline levels in the conditioned copepods were approximately 3 - and 7-fold higher, respectively, than in copepods which had not been cycled.

Abbreviation: FAA free amino acids

* Present address: School of Natural Resources, University of Michigan, Ann Arbor, Michigan 48109, USA
This facilitation in alanine and proline accumulation occurred after 10 and 11 cycles, respectively. Of the increased accumulation in alanine and proline, $7.0 \%$ and $22.5 \%$, respectively, could be accounted for by the higher degree of FAA retention while under hypo-osmotic conditions.

\section{Introduction}

A diversity of organisms, ranging from bacteria through vertebrates (Gilles 1975; Measures 1975; Gilles and Pequeux 1983; Geoffrion and Larochelle 1984) and plants (Stewart and Lee 1974), are known to use intracellular free amino acids (FAA) and other organic solutes for cell volume regulation in response to salinity changes. The typical response among invertebrates is a relatively rapid (hours) increase in the concentration of FAA after hyperosmotic stress, and a more rapid decrease in their concentration upon transfer to a hypo-osmotic medium. The phylogenetic diversity of this phenomenon suggests that selective pressures strongly favor the use of such organic solutes rather than inorganic ions for cellular osmoregulation (Yancey et al. 1982). The advantages of the organic solutes are thought to be due to their stabilizing effect on proteins and other macromolecules, and their enhancement of enzyme activity (Clark 1985). Also, because the organic solutes are less perturbing to enzyme function than inorganic ions, single isozymes can function over the full range of environmental salinities likely to be encountered by a particular species. Hence, a species need not evolve the genetic information for a family of enzymes which can function over a range of ionic concentrations (Yancey et al. 1982). 
The intertidal copepod Tigriopus californicus experiences extensive salinity variation in its natural habitat and has been shown to adjust the concentrations of specific FAA, primarily alanine and proline, in response to salinity changes (Burton and Feldman 1982). Under laboratory conditions, T. californicus is able to reproduce at salinities ranging from 50 to $210 \%$ seawater (Huizinga 1971; Burton and Feldman 1983), and in the field it has been observed at even more extreme salinities (Vittor 1971). Genetic studies have shown that survivability during hyperosmotic stress is at least in part accounted for by the capacity to accumulate FAA (Burton and Feldman 1983).

Studies of acclimation to salinity change have historically been concerned only with acclimation to a new steady-state level (Gilles 1975; Prosser 1986). However, species which are exposed to fluctuating salinities might also experience a second type of acclimation, where past exposure to changing salinity results not in improved function at any single salinity but rather to increased osmoregulatory performance during the stressful transition. Our objective in this study was to determine if exposure of $T$. californicus to a fluctuating salinity regime results in increased rates of FAA accumulation during later episodes of hyperosmotic stress, or a change in the degree of FAA retention during hypo-osmotic stress.

\section{Materials and methods}

Source of animals. T. californicus were collected from Pacific Grove, California, as described by Burton and Feldman (1981). Copepods were kept in the laboratory for several generations in mass cultures at approximately $20^{\circ} \mathrm{C}$. During this time, and in all experiments, the copepods were maintained using artificial seawater ('Instant Ocean').

Experimental protocol. Sixty to 80 adult copepods were randomly assigned to each of six $100 \mathrm{~mm}$ petri dishes. One dish was chosen as the control and maintained at $100 \%$ seawater $\left(1009 \mathrm{mosmol} / \mathrm{kg} \mathrm{H}_{2} \mathrm{O}\right.$ ) for 22 days. The other five dishes were cycled daily between $100 \%$ seawater and either $90,80,70,60$ or $50 \%$ seawater, i.e. $24 \mathrm{~h}$ at high salinity followed by $24 \mathrm{~h}$ at low salinity. Changes in salinity were made by slowly adding either distilled water or a concentrated solution of artificial seawater $(270 \%$ seawater), and then readjusting all treatments to a volume of $25 \mathrm{ml}$. Artificial seawater $(100 \%)$ was also added and removed from the control group to provide conditions similar to the treatments. The experiment was maintained through 11 salinity cycles, during which time the copepods were fed a daily diet of commercial fish food ("Tetra Conditioning Food').

Before the cycling of salinity was begun, one copepod was sampled from each treatment to determine the steady-state level of alanine and proline at $100 \%$ seawater. Thereafter, copepods were sampled from the treatment experiencing the most extreme salinity fluctuation $(50-100 \%$ seawater) $24 \mathrm{~h}$ after transfer to
$100 \%$ seawater to quantify the accumulation of alanine and proline. This was done after $1,2,3,4,6,10$ and 11 cycles, analyzing approximately six animals at each time.

After 10 cycles, all six groups were analyzed for alanine and proline accumulation $24 \mathrm{~h}$ following transfer from their respective low salinity to $100 \%$ seawater. For the control group this was equivalent to a second steady-state observation. After $24 \mathrm{~h}$ at $100 \%$ seawater, all six groups were transferred to $50 \%$ seawater and $24 \mathrm{~h}$ later analyzed for alanine and proline content. Finally, all six groups were transferred to $100 \%$ seawater (11th cycle) and analyzed $24 \mathrm{~h}$ later for alanine and proline accumulation. Approximately six animals were analyzed per group and time for all of the sampling described above. At the conclusion of the experiment, a group of 10 copepods from each treatment was oven-dried $\left(60^{\circ} \mathrm{C}\right)$ and weighed on a Cahn microbalance.

Amino acid determination. Individual copepods were homogenized in $50 \mu 1$ of $80 \%$ ethanol and allowed to evaporate to dryness overnight. FAA were derivatized with dansyl chloride and quantified by HPLC according to the procedure of Burton (1986). Peaks were identified by adding known dansyl amino acids (Sigma; St. Louis, MO) to samples and testing for comigration with sample peaks. All major sample peaks were identified and the amino acids of particular interest, i.e. alanine, proline and taurine, were all well resolved.

Data analysis. Since only a quarter of each copepod preparation was used in the HPLC analysis, each FAA sample was multiplied by four and expressed as pmoles per individual. Because of rapid water loss from live copepods and sampling handling requirements for FAA analysis, obtaining accurate wet or dry weights of individual animals proved impractical. Hence, alanine and proline concentrations were adjusted for copepod body weight using each individual's total taurine content. The overall mean taurine level was divided by each individual's taurine content, and then these values were applied to their respective individual's alanine and proline content. Taurine levels did not change over the course of this experiment $(P>0.30)$, or over the course of an earlier 10-day experiment (Burton and Feldman 1982).

Unless otherwise specified, statistical differences among treatment means were tested by one-way analysis of variance, and the Least Significant Difference (Cochran and Cox 1957).

\section{Results}

\section{Hyperosmotic stress of different magnitude}

The proline and alanine content of T. californicus under steady state conditions $(100 \%$ seawater $)$ were 470.4 pmoles/individual $(\mathrm{SE}=102.3)$ and 186.2 pmoles/individual $(\mathrm{SE}=19.9)$, respectively. Following 10 cycles of salinity fluctuation, alanine and proline levels after $24 \mathrm{~h}$ in $100 \%$ seawater increased in proportion to the magnitude of the osmoregulatory stress (Fig. $1 ; P<0.001$ and 0.005 , respectively). The maximum accumulation of both alanine and proline occurred upon transfer from 60 to $100 \%$ seawater, with additional stress causing little or no further response. Steady-state $(100 \%$ seawater $)$ levels of alanine and proline after 

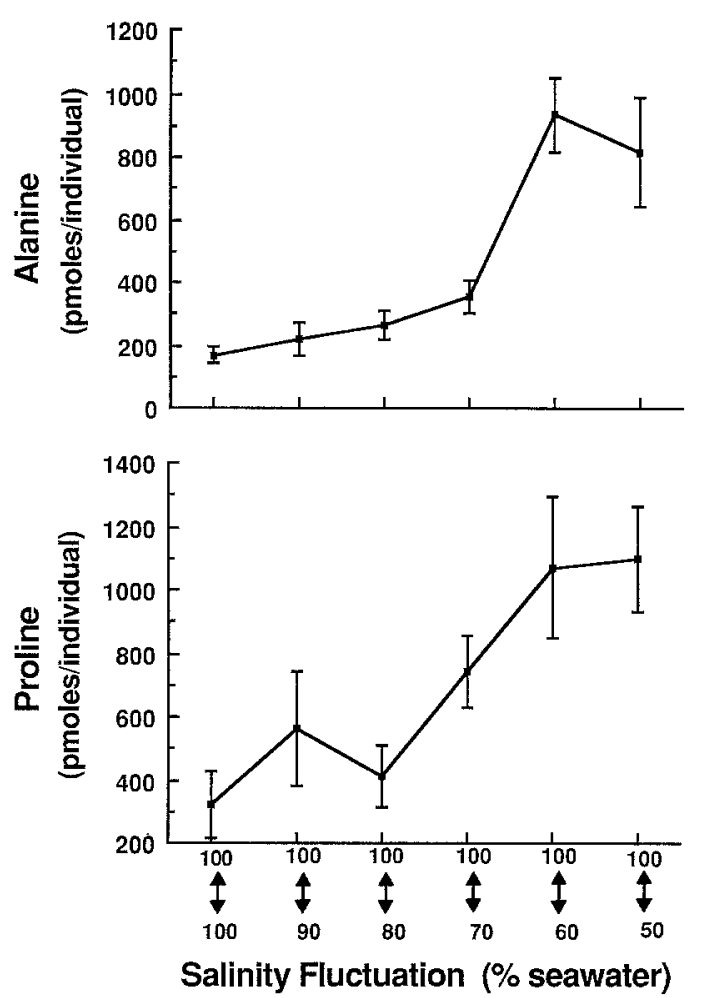

Fig. 1. Total alanine and proline content $(\overline{\mathrm{x}} \pm \mathrm{SE} ; n=6)$ of individual Tigriopus californicus $24 \mathrm{~h}$ after transfer from various low salinities (\% seawater) to $100 \%$ seawater. Each treatment had been previously cycled 9 times between $100 \%$ seawater and its respective hypo-osmotic salinity. Control value is shown as $100-100 \%$ seawater, $n=5$ for $50-100 \%$ seawater treatment

20 days were not significantly different from the start of the experiment $(P>0.05)$.

\section{Hypo-osmotic FAA retention}

After 10 cycles (20 days), all five treatment groups and the steady-state controls were transferred to $50 \%$ seawater - a hypo-osmotic stress which had previously been experienced by only one of the groups. The amount of alanine and proline retained by each treatment after $24 \mathrm{~h}$ is presented in Fig. 2. Animals which had previously been exposed to fluctuating salinity retained a greater amount of alanine $(P<0.001)$ and proline $(P<$ $0.001)$ than the controls. Smaller salinity fluctuations were required to elicit the enhanced retention of alanine $(90-100 \%$ seawater; $P<0.05)$ than the enhanced retention of proline $(60-100 \%$ seawater; $P<0.05, t$-test). The response was most dramatic for the group which had previously experienced the most extreme fluctuation (50-100\% seawater). In this group alanine and proline levels were approximately 10 - and 20 -fold higher, respectively, than the controls.
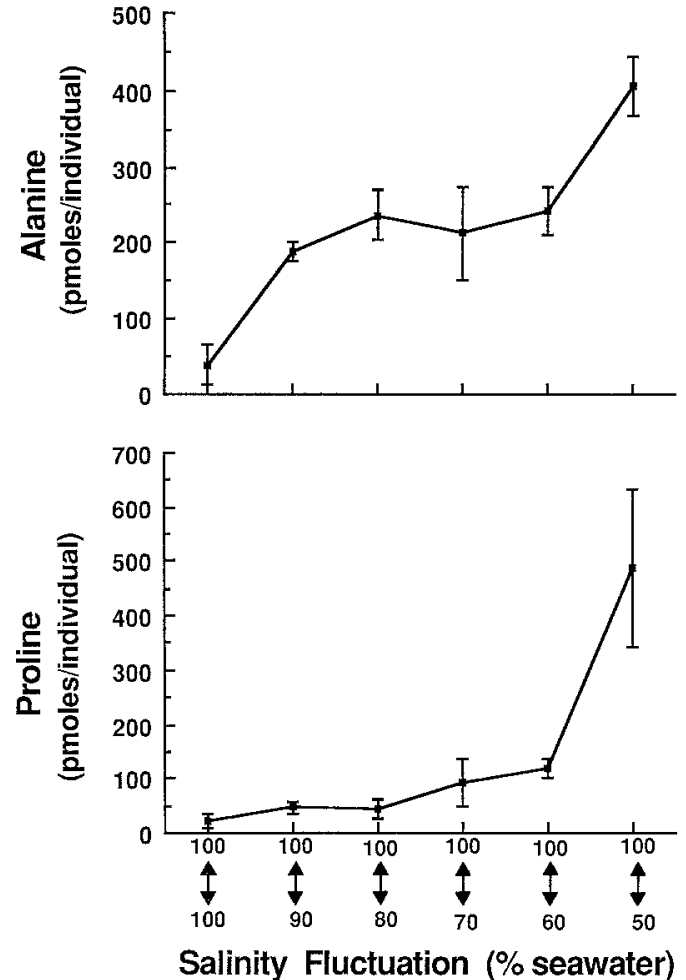

Fig. 2. Remaining alanine and proline content $(\overline{\mathrm{x}} \pm \mathrm{SE} ; n=6)$ of individual $T$. californicus $24 \mathrm{~h}$ after transfer from $100 \%$ to $50 \%$ seawater. The copepods had either been maintained at $100 \%$ seawater for 22 days (100-100\% seawater) or cycled 10 times between $100 \%$ seawater and the hypo-osmotic salinities indicated

\section{Hyperosmotic FAA accumulation}

After $24 \mathrm{~h}$ at $50 \%$ seawater (day 21), all treatment groups and the controls were subjected to the same hyperosmotic stress of $100 \%$ seawater (Fig. 3). The treatment groups which had previously been exposed to fluctuating salinity accumulated significantly more alanine $(P<0.001)$ and proline $(P<$ $0.001)$. In the group which had experienced the most extreme fluctuation (50-100\% seawater) during the previous 10 cycles, alanine and proline levels were approximately 3- and 7-fold higher, respectively, than the controls. The minimum salinity stress required to elicit a significant facilitation in osmotic response was $60-100 \%$ seawater for both alanine $(P<0.05)$ and proline $(P<0.05, t$-test $)$.

\section{Time series}

The treatment group experiencing the most extreme fluctuation $(50-100 \%$ seawater) was also sampled periodically throughout the 22-day experiment (Fig. 4). Alanine levels $24 \mathrm{~h}$ after transfer to $100 \%$ seawater increased as the experiment pro- 

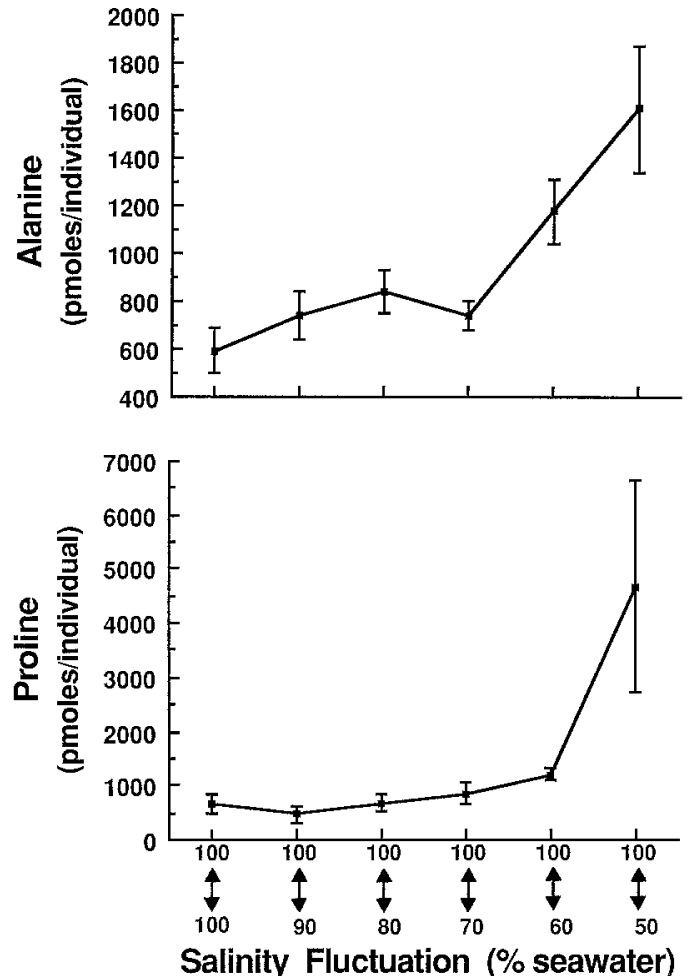

Fig. 3. Total alanine and proline content $(\overline{\mathrm{x}} \pm \mathrm{SE})$ of individual $T$. californicus $24 \mathrm{~h}$ after transfer from $50 \%$ to $100 \%$ seawater. The copepods had either been maintained at $100 \%$ seawater for 22 days (100-100\% seawater) or eycled 10 times between $100 \%$ seawater and the hypo-osmotic salinities indicated. Sample size equals six, except for the control $(n=4)$ and the $50-100 \%$ seawater treatment $(n=5)$

gressed $(P<0.001)$, and by the 11 th cycle had achieved levels approximately 4.5 -fold higher than the control. While proline levels showed a dramatic increase after 11 cycles $(P<0.005)$, the lack of a trend during the earlier samples suggests caution in interpreting this result.

\section{Relative influence of retention and accumulation}

After 21 days, transfer of all groups from 50 to $100 \%$ seawater resulted in higher alanine and proline levels in those treatments which previously experienced salinity fluctuation (Fig. 3). To distinguish the relative contributions of retention while at $50 \%$ seawater versus increased accumulation during hyperosmotic stress, we subtracted from each group's mean accumulated alanine and proline content (Fig. 3) the mean amount of alanine and proline which had been retained while under hypo-osmotic stress (i.e., 50\% seawater; Fig. 2). The difference represents the amount of amino acids accumulated due only to de novo synthesis or protein catabolism (Fig. 5). This component became important, in absolute terms, at a salinity
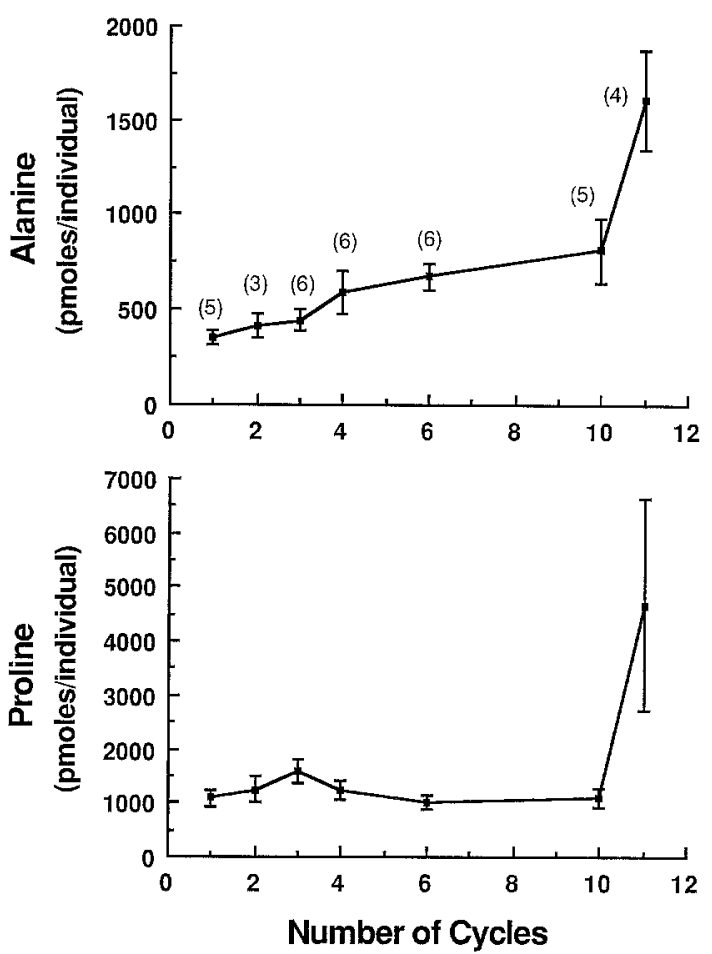

Fig. 4. Total alanine and proline content $(\overline{\mathrm{x}} \pm \mathrm{SE})$ of $T$. californicus $24 \mathrm{~h}$ after transfer from $50 \%$ to $100 \%$ seawater. Values are based on different individuals sampled from a single culture which had been cycled the indicated number of times. Sample sizes are shown in parentheses

fluctuation level of $60-100 \%$ seawater for alanine and $50-100 \%$ seawater for proline. However, of the differences between the control level of FAA at $50 \%$ seawater (Fig. 3) and the amount accumulated by each treatment after $24 \mathrm{~h}$ of hyperosmotic stress, the proportion accounted for by increased retention is relatively small and very similar among the treatments for both alanine $(\overline{\mathrm{x}}=22.5 \%, \mathrm{SE}=$ $1.28)$ and proline $(\overline{\mathrm{x}}=7.0 \%, \mathrm{SE}=1.23)$.

The stress of even the most extreme fluctuation in salinity did not appear to affect the general health of T. californicus. The dry weight of groups of ten animals sampled at the end of the experiment were 100.2, 104.3, 117.7, 105.2, 112.7 and $103.6 \mu \mathrm{g}$ for the control and treatments (in order of increasing stress), respectively. These values are not significantly correlated with the magnitude of stress $(P>0.05)$. The number of copepodites present on day 25 in the control and treatment cultures (in order of increasing stress) were 8, 19, 90, 39, 19 and 201, respectively. These data are not directly comparable since each group began with a different number of adults; however, the very large number of larvae in the most stressed treatment 

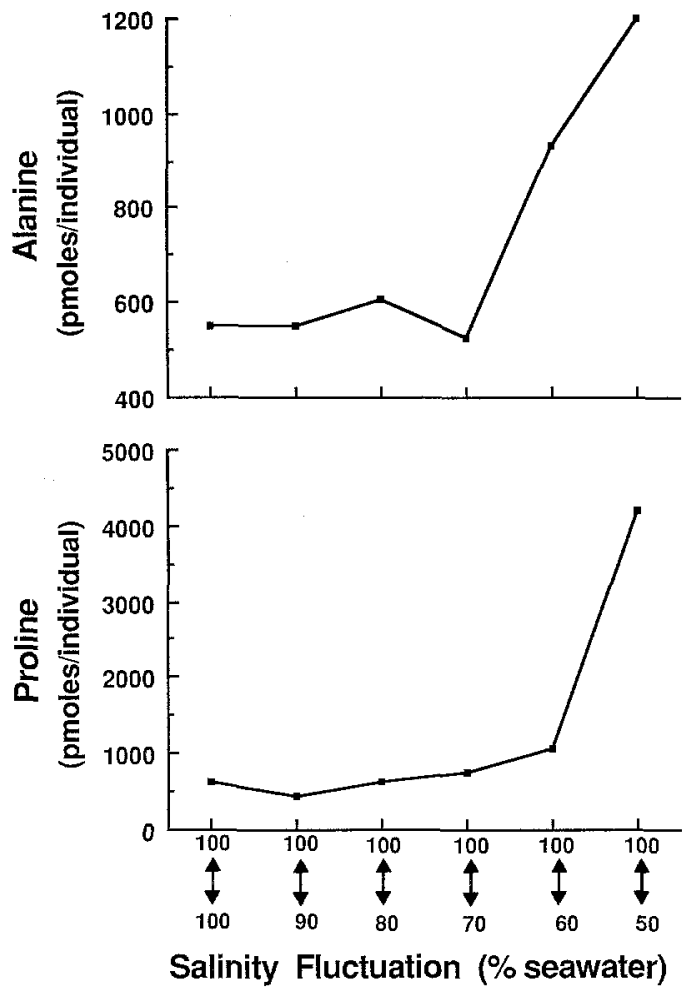

Fig. 5. Total alanine and proline accumulation of individual T. californicus $24 \mathrm{~h}$ after transfer from $50 \%$ to $100 \%$ seawater. The copepods had either been maintained at $100 \%$ seawater for 22 days (100-100\% seawater) or cycled 10 times between $100 \%$ seawater and the hypo-osmotic salinities indicated. The values have been adjusted to account for differences among treatments in amino acid retention under hypo-osmotic conditions

(201) suggests that reproduction is not impaired under a fluctuating salinity regime.

\section{Discussion}

The mechanisms involved in the adjustment of intracellular FAA concentrations in response to changes in environmental salinity are not well understood. One proposed mechanism for which there is some evidence is based on the interaction between inorganic ions and enzyme activity. Under this hypothesis, intracellular concentrations of inorganic ions (in particular $\mathrm{NaCl}$ ) increase during hyperosmotic stress and stimulate the activity of those enzymes which are responsible for the synthesis of FAA and inhibit those which result in their degradation (Gilles 1975; Gilles and Pequeux 1983; Clark 1985). During hypo-osmotic stress, the balance of synthesis and degradation would be such that intracellular FAA levels decline. Alternatively, the decline in FAA levels during hypo-osmotic stress may be due to excretion and/or passive diffusion into the medium.
Copepods exposed to a fluctuating salinity regime retained a greater amount of FAA during hypo-osmotic adjustment than control animals similarly stressed but which had been at constant $100 \%$ seawater. In the group which had experienced the most extreme fluctuation $(50-100 \%$ seawater), alanine and proline concentrations $24 \mathrm{~h}$ after transfer from 100 to $50 \%$ seawater were even higher than the steady-state levels of the $100 \%$ seawater control animals. This dramatic increase in FAA retention contrasts with the rapid decrease in FAA concentration usually observed when T. californicus is exposed to hypo-osmotic stress: alanine and proline concentrations fall to approximately steady-state levels after just $5 \mathrm{~h}$, and typically undershoot their final steady-state levels after $24 \mathrm{~h}$ (Burton and Feldman 1982). Preliminary studies with $T$. californicus indicate that only $5 \%$ of the proline and none of the alanine lost during hypo-osmotic stress can be detected in the medium (in the presence of antibiotic mixture 'AM4' of Provasoli et al. 1959). It would appear, then, that the increased retention is the result of a change in the balance of FAA synthesis and degradation and not a change in the excretion rate. Hypo-osmotic conditions have been shown to stimulate the mitochondrial oxidation of proline in a marine clam (Ballantyne and Storey 1983, 1985); the increased retention of FAA in $T$. californicus may result from a modification of this rate of oxidation.

The increased levels of alanine and proline after hyperosmotic stress in conditioned animals were not simply the result of the increases in FAA retention (discussed above) while at $50 \%$ seawater. In fact, this carry-over accounted for only about 7.0 and $22.5 \%$ of the increased accumulation for proline and alanine, respectively. The increased concentration of FAA, therefore, clearly required a second process as well - increased rates of accumulation upon transfer to $100 \%$ seawater. Evidence that at least some of the increase in FAA levels during hyperosmotic stress is due to de novo synthesis was provided by Burton (1986). Using ${ }^{14} \mathrm{C}$ bicarbonate as a tracer, it was shown that incorporation of label occurs into both alanine and proline during hyperosmotic stress. In the present study, a threshold of salinity stress seemed to be necessary to elicit the enhanced rate of FAA synthesis (Fig. 5). For alanine this threshold occurred at a salinity fluctuation of $60-100 \%$ seawater, and for proline a $50-100 \%$ seawater fluctuation cycle was required.

Acclimation to fluctuating salinity may result in modifying the processes responsible for producing FAA (hyperosmotic phase) and/or those which result in the elimination of FAA (hypo-osmotic 
phase). The results of the present study indicate that exposure to fluctuating salinity modifies FAA metabolism in the direction of increased accumulation, suggesting that the osmoregulatory adjustments made during hyperosmotic stress are more amenable to regulation than those which occur during hypo-osmotic stress. After transfer to hypoosmotic conditions, we observed very different patterns of retention for alanine and proline (Fig. 2). That is, acclimation to a fluctuating salinity of just $90-100 \%$ seawater increased the retention of alanine during hypo-osmotic stress, whereas a more severe fluctuating salinity regime of $60-100 \%$ seawater was needed to elicit the increased retention of proline. This also indicates that the mechanism responsible for increased FAA retention is not simply a non-selective decrease in permeability, but is rather the result of an amino acid-specific change in metabolism.

The increased capacity to synthesize FAA during hyperosmotic stress in conditioned animals may also explain the apparently higher retention while under hypo-osmotic stress. If the metabolic pathways involved in FAA synthesis are accelerated by repeated salinity fluctuation, then the balance of synthesis and degradation would be shifted to result in higher concentrations of alanine and proline even under hypo-osmotic conditions. The similarity in response, among the treatments, for FAA retention (Fig. 2) and facilitated accumulation (Fig. 3) strongly suggests that this is the case. Furthermore, for each of the amino acids studied, the proportion of facilitated accumulation accounted for by increased retention was almost invariant among the treatments. These results, therefore, support the view that both the accumulation and loss of FAA following a change in salinity can be regulated at the point of FAA synthesis.

Inorganic ions have been implicated in the regulation of short-term FAA accumulation in invertebrates by direct interaction with existing enzymes. The key enzyme in glutamate synthesis, glutamate dehydrogenase (GDH), is one of those whose activity has been shown to be stimulated by increased $\mathrm{NaCl}$ concentration (Schoffeniels and Gilles 1963; Gilles 1974). Since glutamate is a precursor of proline, the acceleration of proline synthesis during hyperosmotic stress in $T$. californicus may therefore result from the action of either $\mathrm{NaCl}$ or other ions on GDH activity. This would result in the diversion of $\alpha$-ketoglutarate from Krebs-cycle oxidation to the synthesis of FAA. We have indeed found a decrease in the oxygen consumption rate of $T$. californicus upon transfer to a hyperosmotic medium (unpublished data). While it is possible that the facilitation in FAA accumulation observed in this study is the result of a general increase in metabolic rate, the different responses found for alanine and proline suggest that more specific mechanisms are operating. We are not aware of any study which has demonstrated a difference in the concentration of enzymes involved in FAA synthesis between animals acclimated to low and high salinity (Chaplin et al. 1965; Gilles and Pequeux 1983). It is possible, however, that the longer-term stimulus of repeated hyperosmotic episodes (as studied here) would result in the increased synthesis of enzymes involved in alanine and proline metabolism. This type of osmotic regulation in enzyme synthesis has recently been reported among prokaryotes (Landfald and Strom 1986; Styrvold et al. 1986).

To assess the adaptive significance to $T$. californicus of facilitation in FAA accumulation we must consider the actual contribution of alanine and proline to cellular osmolarity during salinity stress. While it was not possible to determine this directly, an estimate of their contribution for a given set of conditions was obtained as follows. By weighing groups of copepods (six groups of ten), we estimated the average wet weight of an adult copepod to be $34.9 \mu \mathrm{g}(\mathrm{SE}=0.99)$. From this value we subtracted the mean dry weight per individual $(10.7 \mu \mathrm{g})$, as estimated in the present study, and an additional $5.1 \mu \mathrm{g}$ to account for the large gut volume of T. californicus (T. Merchant 1978, unpublished manuscript; Hopkins Marine Station Library). Reported values for the extracellular fluid volume of crustaceans range from 17 to $33 \%$ of body weight (Prosser 1973). Using an intermediate value of $25 \%$, we estimated intracellular water content at $14.3 \mu \mathrm{g}$ per individual. If we consider typical alanine and proline concentrations following hyperosmotic stress (1000 pmoles/individual each; Fig. 1), we calculate their combined osmolarity to be $139.9 \mathrm{mosmol} / \mathrm{kg}$. Although there is considerable variability in response, further calculations such as these indicate that facilitation increased the contribution of alanine and proline from approximately 9.0 to $42 \%$ of total osmolarity. These percentages are based on the assumption that $T$. californicus is iso-osmotic at $100 \%$ seawater, as is the case for the majority of marine crustaceans (Gilles and Pequeux 1983).

The adaptive significance to $T$. californicus of facilitation in FAA accumulation seems clear. When occurring in a habitat where the salinity often fluctuates, conditioned individuals will be able to respond more quickly and completely to hyperosmotic stress. Such animals would also, presum- 
ably, be able to tolerate higher salinity levels should they occur. In addition, greater FAA concentrations would require less dependence on inorganic ions - thus minimizing their perturbing effect on enzyme function. The small increase in retention of FAA under hypo-osmotic conditions would not appear to be a disadvantage since it would not result in intracellular solute concentrations greater than that of the environment.

Initially, we expected that the exposure of $T$. californicus for three weeks to a repeated daily osmoregulatory challenge would be very stressful. However, based on the weight of copepods at the end of the experiment and the number of copepodites produced, it appears that $T$. californicus is able to cope with such an extreme fluctuating salinity regime. There was some indication that reproduction may even be enhanced by fluctuating salinities. The additional energy required for osmoregulation was probably obtained by increasing the rate of food ingestion - food was provided ad libitum. However, the growth and survival of $T$. californicus under natural conditions, where food might be limiting, may be negatively affected by the severe fluctuating salinity regime used in this study.

The physiological response of $T$. californicus and other invertebrates to changes in salinity have recently been used as a model to understand the links between allozyme polymorphism and organismal fitness (Hilbish et al. 1982; Burton and Feldman 1983). These studies have shown that individual variability in physiological response to salinity stress (including survival) is in part due to genetic polymorphisms. The present study demonstrates that variability between individuals (and populations) in physiological response to salinity changes can also be due to the organism's salinity history.

Acknowledgement. This research was supported by grants from the National Science Foundation to RSB (BSR-8415819, BSR8741308).

\section{References}

Ballantyne JS, Storey KB (1983) Mitochondria from the ventricle of the marine clam, Mercenaria mercenaria: substrate preferences and effects of $\mathrm{pH}$ and salt concentration on proline oxidation. Comp Biochem Physiol 76 B:133-138

Ballantyne JS, Storey KB (1985) Solute effects on mitochondrial respiration: the kinetics of proline oxidation by mitochondria from the ventricle of the marine clam Mercenaria mercenaria. Comp Biochem Physiol 81 B:777-780

Burton RS (1986) Incorporation of ${ }^{14} \mathrm{C}$-bicarbonate into the free amino acid pool during hyperosmotic stress in an intertidal copepod. J Exp Zool 238:55-61

Burton RS, Feldman MW (1981) Population genetics of Tigriopus californicus: II. Differentiation among neighboring populations. Evolution 35:1192-1205
Burton RS, Feldman MW (1982) Changes in free amino acid concentrations during osmotic response in the intertidal copepod Tigriopus californicus. Comp Biochem Physiol 73 A:441-445

Burton RS, Feldman MW (1983) Physiological effects of an allozyme polymorphism: glutamate-pyruvate transaminase and response to hyperosmotic stress in the copepod Tigriopus californicus. Biochem Genet 21:239-251

Chaplin AE, Huggins AK, Munday KA (1965) Ionic effects on glutamate dehydrogenase activity from beef liver, lobster muscle and crab muscle. Comp Biochem Physiol 16:49-62

Clark ME (1985) The osmotic role of amino acids: discovery and function. In: Gilles R, Gilles-Baillien M (eds) Transport processes, iono- and osmoregulation. Springer, Berlin Heidelberg New York, pp $412-423$

Cochran WG, Cox GM (1957) Experimental designs. Wiley, New York

Geoffrion Y, Larochelle J (1984) The free amino acid contribution to osmotic regulation in Acanthamoeba castellanii. Can J Zool 62:1954-1959

Gilles R (1974) Studies on the effect of $\mathrm{NaCl}$ on the activity of Eriocheir sinensis glutamate dehydrogenase. Int $\mathbf{J}$ Biochem 5:623-628

Gilles R (1975) Mechanisms of ion and osmoregulation. In: Kinne O (ed) Marine ecology, vol 2, part 1. Wiley, London, pp 259-347

Gilles R, Pequeux A (1983) Interactions of chemical and osmotic regulation with the environment. In: Vernberg FJ, Vernberg WB (eds) The biology of Crustacea, vol 8. Environmental adaptations. Academic Press, New York, pp 109-177

Hilbish TJ, Deaton LE, Koehn RK (1982) Effect of an allozyme polymorphism on regulation of cell volume. Nature 298:688-689

Huizinga HW (1971) Cultivation, life history and salinity tolerance of the tidepool copepod, Tigriopus californicus Baker 1912, in artificial seawater. Trans Ill State Acad Sci $64: 230-236$

Landfald B, Strom AR (1986) Choline-glycine betaine pathway confers a high level of osmotic tolerance in Escherichia coli. J Bacteriol 165:849-855

Measures JC (1975) Role of amino acids in osmoregulation of non-halophilic bacteria. Nature 257:398-400

Prosser CL (1973) Circulation of body fluids. In: Prosser CL (ed) Comparative animal physiology. Saunders, Philadelphia, pp 822-856

Prosser CL (1986) Adaptational biology: Molecules to organisms. Wiley, New York, $784 \mathrm{pp}$

Provasoli L, Shiraishi K, Lance JR (1959) Nutritional idiosyncrasies of Artemia and Tigriopus in monoxenic culture. Ann New York Acad Sci 77:250-261

Schoffeniels E, Gilles R (1963) Effect of cations on the activity of L-glutamic acid dehydrogenase. Life Sci 2:834-839

Stewart GR, Lee JA (1974) The role of proline accumulation in halophytes. Planta 120:279-289

Styrvold OB, Falkenberg P, Landfald B, Eshoo MW, Bjornsen T, Strom AR (1986) Selection, mapping, and characterization of osmoregulatory mutants of Escherichia coli blocked in the choline-glycine betaine pathway. J Bacteriol $165: 856863$

Vittor BA (1971) Effects of the environment on fitness-related life history characteristics in Tigriopus californicus. PhD thesis, University of Oregon, Eugene, Oregon

Yancey PH, Clark ME, Hand SC, Bowlus RD, Somero GN (1982) Living with water stress: evolution of osmolyte systems. Science $217: 1214-1222$ 